\title{
The MEFISTO and WPT Electric Field Sensors of the Plasma Wave Investigation on the BepiColombo Mio Spacecraft
}

\author{
Measurements of Low and High Frequency Electric Fields \\ at Mercury
}

\section{T. Karlsson ${ }^{1}$ (D) Y. Kasaba ${ }^{2}$ - J.-E. Wahlund ${ }^{3}$ P. Henri ${ }^{4,5}$ - L. Bylander ${ }^{1}$ - W. Puccio ${ }^{3}$. S.-E. Jansson ${ }^{3}$ - L. Åhlen ${ }^{3}$ - E. Kallio ${ }^{6}$ H. Kojima ${ }^{7}$ A. Kumamoto ${ }^{8}$ - K. Lappalainen ${ }^{9}$. B. Lybekk ${ }^{10}$ - K. Ishisaka ${ }^{11}$ - A. Eriksson ${ }^{3}$ - M. Morooka ${ }^{3}$}

Received: 28 December 2019 / Accepted: 30 October 2020 / Published online: 12 November 2020

(C) The Author(s) 2020

\begin{abstract}
This paper describes the design of MEFISTO (Mercury Electric Field In-Situ Tool) and WPT (Wire Probe Antenna) electric field sensors for Plasma Wave Investigation (PWI) on the BepiColombo Mio spacecraft (Mercury Magnetospheric Orbiter, MMO). The two sensors will enable the first observations of electric fields, plasma waves and radio waves in and around the Hermean magnetosphere and exosphere. MEFISTO and WPT are dipole antennas with $31.6 \mathrm{~m}$ tip-to-tip length. Each antenna element has a spherical probe at each end of the wire (15 m length). They are extended orthogonally in the spin plane of the spacecraft and enable measurements of the electric field in the frequency range of DC to $10 \mathrm{MHz}$ by the connection to two sets of receivers, EWO for a lower frequency range and
\end{abstract}

The BepiColombo mission to Mercury

Edited by Johannes Benkhoff, Go Murakami and Ayako Matsuoka

T. Karlsson

tomas.karlsson@ee.kth.se

1 Space and Plasma Physics, KTH Royal Institute of Technology, 10405 Stockholm, Sweden

2 Planetary Plasma and Atmospheric Research Center, Graduate School of Science, Tohoku University, Sendai, Miyagi 980-8578, Japan

3 Swedish Institute of Space Physics, Box 537, 75121 Uppsala, Sweden

4 LPC2E, CNRS, Orléans, France

5 Laboratoire Lagrange, CNRS, Observatoire de la Côte d'Azur, Université Côte d'Azur, Nice, France

6 Finnish Meteorological Institute, Space Research Unit, Helsinki, Finland

7 Research Institute for Sustainable Humanosphere, Kyoto University, Uji, Kyoto 611-0011, Japan

8 Department of Geophysics, Graduate School of Science, Tohoku University, Sendai, Miyagi 980-8578, Japan

9 University of Oulu, Oulu, Finland

10 Department of Physics, University of Oslo, Oslo, Norway

11 Department of Electronics and Informatics, Toyama Prefectural University, Imizu, Toyama 939-0398, Japan 
SORBET for higher frequencies. In the initial operations after the launch (20 Oct. 2018), we succeeded to confirm the health of both antennas and to release the launch lock of the WPT. After Mercury orbit insertion planned at the end of 2025, both sensors will be fully deployed and activate full operations of the PWI electric field measurements.

Keywords Electric fields · Plasma waves - Spacecraft potential · Electron density and temperature · MEFISTO - Wire Probe Antenna (WPT) · Plasma Wave Investigation (PWI) . Mio $\cdot$ Mercury Magnetospheric Orbiter (MMO) $\cdot$ BepiColombo

\section{Introduction}

The BepiColombo project is a Euro-Japanese joint mission for the investigation of Mercury, the innermost planet in our solar system (Benkhoff et al. 2010, 2020, this issue). The Mio (Mercury Magnetospheric Orbiter (MMO)) spacecraft is a part of this project (Hayakawa et al. 2004; Yamakawa et al. 2008; Murakami et al. 2020, this issue), and will enable a comprehensive study of the Hermean environment, which is strongly influenced by the Sun, by combined observations with the Mercury Planetary Orbiter (MPO) (Milillo et al. 2010, 2020, this issue). The spacecraft was successfully launched on October 2018 as a unified cruise composite system, and is presently on a long cruise with the arrival to Mercury planned at the end of 2025 .

The Plasma Wave Investigation (PWI) aboard the Mio spacecraft will provide the first opportunity to measure electric field, plasma waves, and radio waves in electric field (DC $10 \mathrm{MHz})$ and magnetic field $(0.1 \mathrm{~Hz}$ to $640 \mathrm{kHz})$ around the Hermean magnetosphere and exosphere (Kasaba et al. 2010, 2020).

For the electric field measurement, the PWI has two pairs of wire dipole antennas, MEFISTO (Mercury Electric Field In-Situ Tool) and WPT (Wire Probe Antenna) (Fig. 1). Both sensors with their deployment units are orthogonally installed on the outer side of the spacecraft lower deck. Each dipole antenna is a pair of top-hat wire probe antennas, with a sphere attached at the end of the wire (length: $15 \mathrm{~m}$ ), and its total length after deployment is $31.6 \mathrm{~m}$ tip-to-tip.

Figure 2 shows a block diagram of the PWI system. The sensor signals are fed to two receivers, the EWO (EFD, WFC, and OFA) for the lower frequency range and SORBET (Spectroscopie Ondes Radio and Bruit Electrostatique Thermique) for the higher frequency range. EWO consists of the EFD (Electric Field Detector), covering less than $32 \mathrm{~Hz}$ and WFC/OFA (WaveForm Capture and Onboard Frequency Analyzer) covering $10 \mathrm{~Hz}$ to 20/120 kHz. SORBET covers frequencies from $2.5 \mathrm{kHz}$ to $10 \mathrm{MHz}$. MEFISTO is also used for the active impedance measurement by $\mathrm{AM}^{2} \mathrm{P}$ (Active Measurement of Mercury's Plasma). Descriptions of PWI, including its overall design and specifications, together with EWO and SORBET, are detailed in Kasaba et al. (2020), while Yagitani et al. (2020, this issue) covers the Search Coil Magnetometer.

MEFISTO (Fig. 1a and b) is provided from the Swedish and Nordic teams. Its design is based on principles with heritage from the EFW antenna system aboard the Cluster spacecraft (Gustafsson et al. 2001), and has a significantly reduced mass compared to conventional units thanks to a newly developed deployment mechanism. Its sensor part (MEFISTO-S) consists of a deployable boom, a pre-amplifier box, and a conductive short wire with a sphere at the end. The pre-amplifier box is attached to the end of the deployable boom, and the short wire with the sphere attached is deployed from the probe. The antenna is designed to provide good frequency response below $3 \mathrm{MHz}$. Two MEFISTO-S booms are electrically 

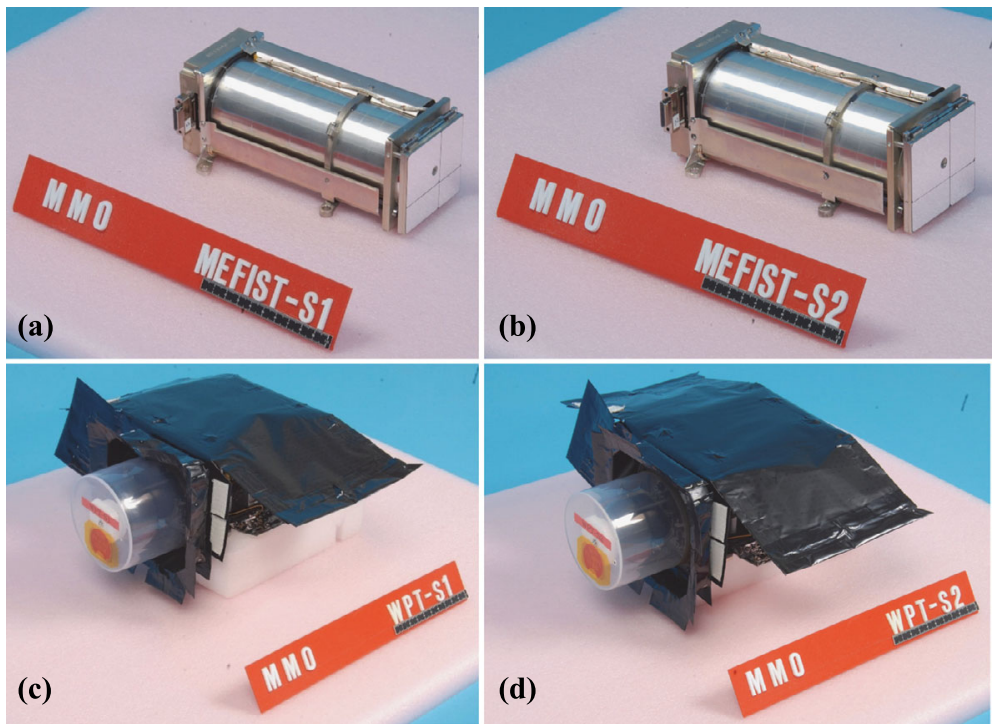

Fig. 1 Pictures of PWI wire antenna booms at the shipment to the system in 2012. (a) MEFISTO-S1, (b) MEFISTO-S2, (c) WPT-S1, (d) WPT-S2

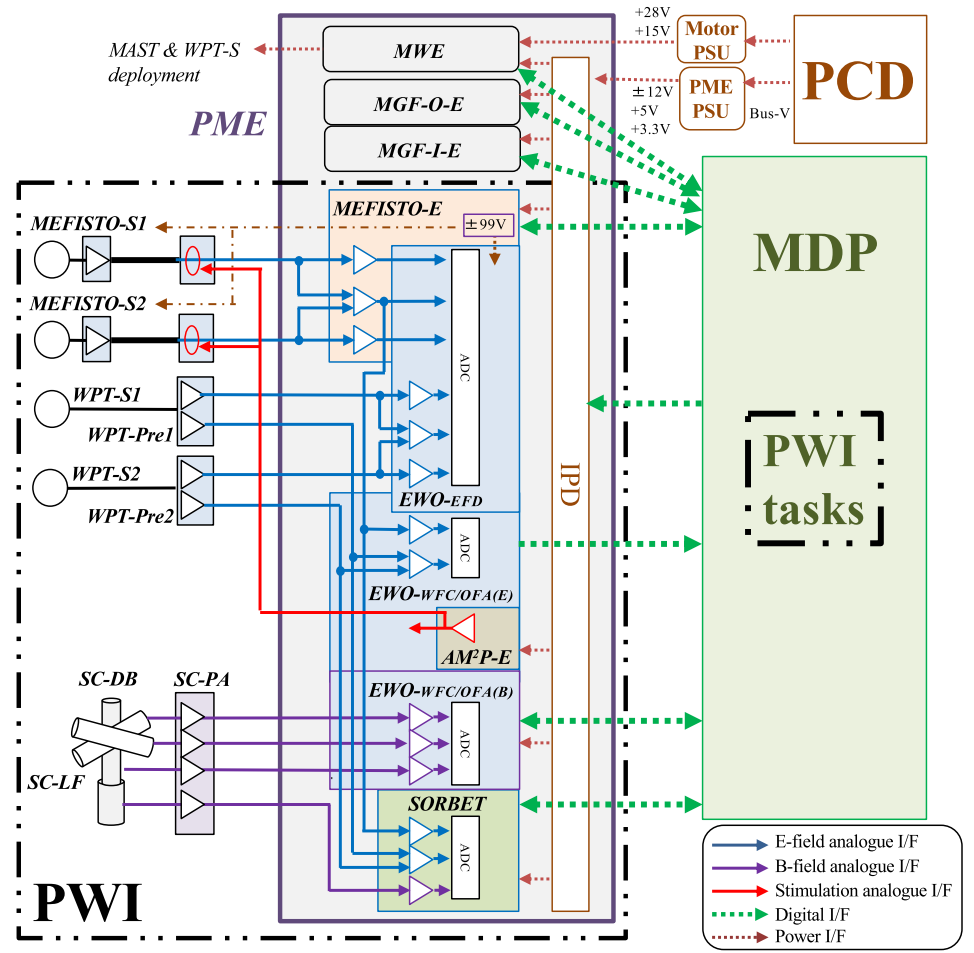

Fig. 2 MEFISTO and WPT in the PWI system. Characteristics of the I/F lines are summarized in the drawing. The deployment of MEFISTO and WPT are controlled by MEFISTO-E and MAST-WPT-E in the PME chassis. (From Kasaba et al. 2020) 
connected to the control electronics (MEFISTO-E) which is installed in the PWI-MGF Electrical box (PME). MEFISTO-E provides the deployment controls and status monitoring, but also contributes to the first-stage amplifications of signals, and controls the bias current and guard voltage, and the power supply to the MEFISTO-S pre-amplifier.

The WPT (Wire-Probe ANTenna) (Fig. 1c and d) is provided from the Japanese team. Its design is based on principles with heritage from the PANT antenna system aboard the Geotail spacecraft (Tsuruda et al. 1994; Matsumoto et al. 1994). An almost identical model, the PWE WPT, is on board the Arase spacecraft and has performed well (Kasahara et al. 2018; Kasaba et al. 2017). Its sensor part (WPT-S) consists of a conductive wire with a sphere at the end of the long wire. The signal picked up by this antenna is directly fed to its pre-amplifier (WPT-Pre) which is located on the inner side of the spacecraft lower deck. Because of the benefit of the long wire (see e.g. Ergun et al. 2001; Kasaba et al. 2017), the WPT has its advantage in higher frequency measurement up to $10 \mathrm{MHz}$. The deployment lines of the two WPT-S booms are electrically connected to the drive and control electronics (MAST-WPT-E) which is installed in the PWI-MGF Electrical box (PME). MAST-WPT-E provides the deployment controls and status monitoring of WPT-S. In addition, the signal lines of the WPT-S booms are connected to the EWO receiver which is installed in the PME chassis through WPT-Pre. The EWO contributes to the first-stage amplification of the signals and the bias current controls for WPT-S.

On the Mio spacecraft, the four PWI wire booms, WPT-S1, MEFISTO-S1, WPT-S2, and MEFISTO-S2, are orthogonally installed at the outer side of the lower deck, perpendicular to the side panel. Figure 3 describes their geometry in (a) the external view, (b) the view along the Z-axis (spin axis), and (c) a schematic view. In the spacecraft coordinates, WPT-S1, MEFISTO-S1, WPT-S2, and MEFISTO-S2 are extended in the spin plane toward $+\mathrm{X},+\mathrm{Y}$, $-\mathrm{X}$, and $-\mathrm{Y}$, respectively. The spin axis of Mio is parallel to the Z-axis, almost perpendicular to the Sun-Earth line. For both of them, their spheres and wires will be extended through a hole in the side panel in order to prevent penetration of the intense solar heat flux into the spacecraft. Ideally, for the electric field and potential measurement, the probes should be separated by a distance longer than Debye length in order to reduce the disturbances from the spacecraft potential. However, the wire length is limited by spin rate, moment of inertia, and mass. Although the expected Debye length in the Hermean environment can be as large as $10-100 \mathrm{~m}$, the wire length has been set at $15 \mathrm{~m}$, as a compromise. The total length after deployment is $31.6 \mathrm{~m}$ tip-to-tip.

The DC electric field measurement by both antennas is based on the principle of doubleprobe measurement. This concept is identical to that of an ideal voltmeter; the measurement of the difference in plasma potential between two probes. If we can ensure that the deviations of probe potentials from the surrounding plasma potentials is small, the plasma electric field along the antenna can be derived as (potential difference [V])/(distance of two probes [m]). The potential of a conductive material in a plasma under the Hermean strong solar flux is determined by the balance between the photoelectron inflow current (or photoelectron outflow), $I_{p h}$, and the ambient electron outflow current (or ambient electron inflow) from the surrounding plasma, $I_{e}$. When the probe potential is close to zero (or close to the ambient plasma potential), $I_{p h}\left(5-50 \mathrm{nA} / \mathrm{cm}^{2}\right)$ is much larger than $I_{e}\left(0.1-10 \mathrm{nA} / \mathrm{cm}^{2}\right)$ in the low plasma density $\left(0.1-100 / \mathrm{cm}^{2}\right)$ of the solar wind, magnetosphere, and exosphere around Mercury. Under this condition, conductive materials become positively charged, and the inflow electron current is balanced by the reduced photoelectron outflow. In this condition, the probe potential deviates from the plasma potential. Under this 'floating' condition, the resistance of the probe relative to the ambient plasma is larger than several hundred $\mathrm{M} \Omega$.

Because of this high resistance, the probe potential is highly sensitive to even small changes of ambient electron inflow associated with small variations of electron density 


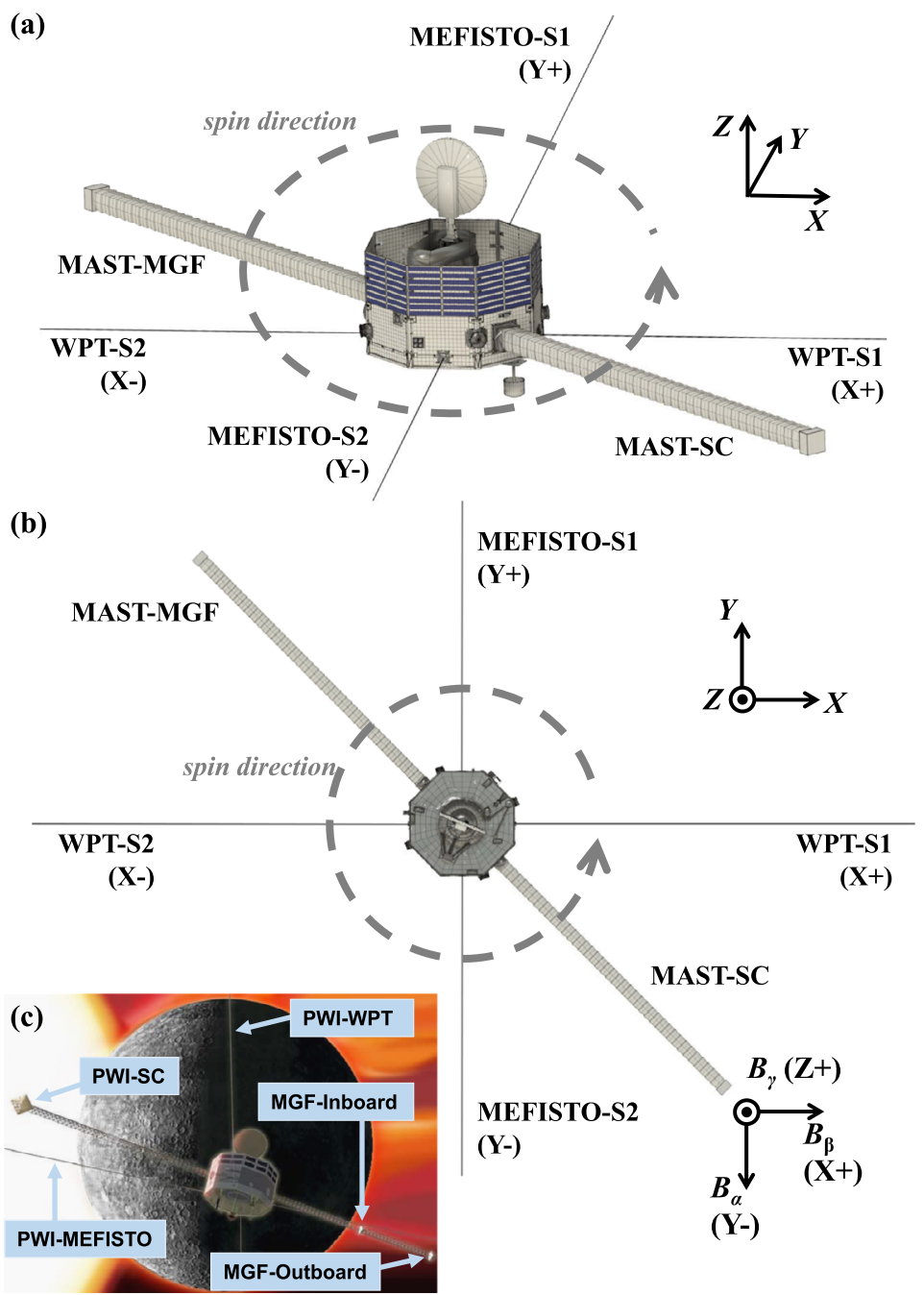

Fig. 3 Location of MEFISTO and WPT sensors on the Mio spacecraft. (a) External view, (b) view along the spin axis $(+Z)$, and $(\mathbf{c})$ a schematic view. The spacecraft coordinates and the direction of the spacecraft spin rotation (spin rate: $4-5 \mathrm{sec}$ ) are also shown. (From Kasaba et al. 2020)

and/or temperature. In order to reduce the potential difference of the probe and the resistance to ambient plasma, an outflow bias current $I_{b}$ from the probe (or inflow of bias electrons to the probe) is additionally fed with the absolute amount less than but close to $I_{p h}$, to compensate the photoelectron outflow from the probe. In this condition, the photoelectron outflow $I_{p h}$ is closely balanced to the inflow of ambient electrons and bias current electrons $I_{e}+I_{b}$, and the probe potential can be more stable and close to the ambient plasma potential. The bias current is set so that the sensor operates in the region close to the highest gradient of the photoelectron current to the S/C potential (i.e., lowest impedance) (Pedersen et al. 1998). Using such biased probes, the resistance to ambient plasma becomes several to $100 \mathrm{M} \Omega$, and we can measure the electric field of the ambient plasma with the accuracy on the order of $\mathrm{mV} / \mathrm{m}$ or better. 
By biasing the probe we can also measure the floating spacecraft potential with respect to the ambient plasma. Because the biased probe has its potential close to that of the ambient plasma, the negative of the probe potential from the spacecraft ground can be used as the spacecraft potential, and can be used as an indirect indicator of the surrounding electron density and temperature variations with higher time resolutions than other measurement techniques (e.g. Laakso and Pedersen 1998). This information is also useful for the data correction of lower-energy particle measurements affected by the spacecraft floating potential.

The floating potential measurements are particularly useful when combined with independent absolute density measurements, such as those to be performed by $\mathrm{AM}^{2} \mathrm{P}$ through measurements of the MEFISTO mutual impedance, to extract high cadence and fully calibrated plasma density time series after a careful cross-calibration process, such as it has been done, e.g., for Rosetta with the Mutual Impedance Probe RPC-MIP and the dual Langmuir Probe RPC-LAP instruments (Heritier et al. 2017; Breuillard et al. 2019).

By biasing the MEFISTO-S and WPT-S antennas, PWI EWO-EFD can measure the electric field in the frequency range of DC to $32 \mathrm{~Hz}$ with dynamic amplitude range from -500 to $+500 \mathrm{mV} / \mathrm{m}$ and with a resolution of $0.015 \mathrm{mV} / \mathrm{m}$. This range is enough to cover the expected fields in the exosphere, magnetosphere and solar wind around Mercury (e.g. Blomberg et al. 2006). In addition, PWI EWO-EFD can also measure the spacecraft potential with a dynamic range from -100 to $+100 \mathrm{~V}$ and with a resolution of $3 \mathrm{mV}$, which will cover the potentials produced by the expected electron densities and temperatures around Mercury.

The basic principle of higher frequency electric field measurement is identical to that of a normal monopole or dipole antenna. The capacitive coupling of the wire antenna (conductive wire + sphere at the end) in vacuum can be obtained as $C_{\text {wire }}=l /(120 c \ln (2 l / a))$ and $C_{\text {sphere }}=4 \pi \epsilon_{0} R$, where $C_{\text {wire }}$ is the capacitance of the conductive wire, $c$ is the speed of light, $l$ is the length of the conductive wire, $a$ is the radius of the wire, $C_{\text {sphere }}$ is the capacitance of the sphere, $\epsilon_{0}$ is the electric permittivity, and $R$ is radius of the sphere. When used in combination with EWO (WFC/OFA) and SORBET, WPT-S and MEFISTO-S will measure the electric field in the ranges $10 \mathrm{~Hz}-10 \mathrm{MHz}$ and $10 \mathrm{~Hz}-3 \mathrm{MHz}$, respectively. We note that in the frequency lower than plasma frequencies, those characteristics are affected by ambient plasma parameters. Careful calibration will be needed by the PWI team.

Finally, $\mathrm{AM}^{2} \mathrm{P}$ will provide both self and mutual impedance measurements of the MEFISTO-S sensor in the $[0.128 \mathrm{kHz}-143.36 \mathrm{kHz}]$ frequency range to independently extract both the absolute electron density and the electron temperature. To this purpose, MEFISTO-S is used in three different configurations: (i) as a double sphere antenna, with an oscillating current being injected by $\mathrm{AM}^{2} \mathrm{P}$ onto the spherical probes MEFISTO-S through a resistor-capacitor network, while the voltage is simultaneously measured at the same frequency between the same spherical probes; (ii) as a double wire antenna, with an oscillating voltage being applied to the external shield of the MEFISTO deployment cables, while the current flowing through the same wire(s) is simultaneously measured at the same frequency; (iii) as a quadrupolar antenna formed by two spherical antennas (the MEFISTO-S sensors) and two wire antennae (the wire shields of MEFISTO deployment cables), with an oscillating voltage being applied to the wire shields, while the induced voltage is simultaneously measured between the two spherical probes at the same frequency. In these three configurations, $\mathrm{AM}^{2} \mathrm{P}$ will have access to, respectively, (i) the self-impedance of the MEFISTO-S double-sphere probe antenna, (ii) the self-impedance of the MEFISTO deployment cable used as a double wire-shield antenna, and (iii) the mutual impedance of the equivalent quadrupolar MEFISTO antennas composed of the MEFISTO double wire-shield and double-sphere probe antennae. 


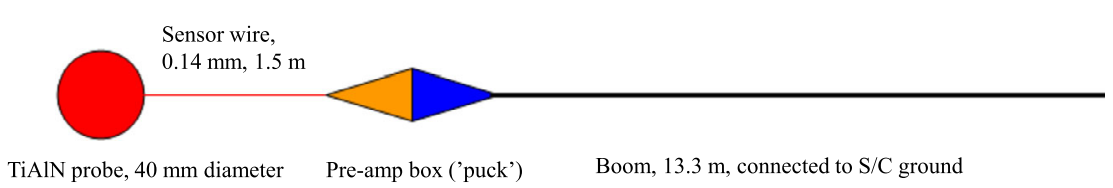

Fig. 4 MEFISTO sensor configuration. Adapted from Blomberg et al. (2006)

The following sections describe the detailed design of the MEFISTO and WPT systems. By the combination of MEFISTO and WPT, the PWI measurements can investigate the Hermean environment and the inner heliosphere in combination with to the magnetic field measurement by MGF and PWI SC-LF and SC-DB.

\section{MEFISTO}

\subsection{Basic Design}

The MEFISTO (Mercury Electric Field In Situ TOol) double-probe electric field instrument (Blomberg et al. 2006), is based on heritage from the Cluster electric field instrument (Electric Fields and Waves) (Gustafsson et al. 2001; Khotyaintsev et al. 2014). Following the design of the EFW instrument, the pre-amplifiers are located in a so-called 'puck', close to the probe. The small distance of the pre-amplifier to the probe allows high-quality measurements in a wide frequency range. At the same time, having the pre-amplifiers some distance away from the probe, allows the probe to be attached to it by a very thin single wire, the sensor wire, which minimizes the disturbances around the probe. The puck is connected to the spacecraft with a thicker, multi-conductor boom cable.

Another important reason for this design is that separating the measurement probe from the pre-amplifiers allowed a choice of probe surface material optimizing the electrical contact with the surrounding plasma, at the same time as choosing a puck surface material providing an acceptable thermal environment for the pre-amplifier electronics. Because of the proximity to the Sun, and the thermal radiation from Mercury, this is of crucial importance.

MEFISTO consists of two boom units at the outer side of the lower deck, MEFISTO-S, with a sphere, sensor wire, pre-amplifier (inside a puck), multi-conductor boom cable, and a deployment unit, and MEFISTO-E in the PME chassis, with motor drive and electronics for gain, bias, potentials of puck surfaces, and calibration.

\subsection{MEFISTO-S}

Figure 4 illustrates the MEFISTO sensor configuration. The tip-to-tip distance is $31.6 \mathrm{~m}$ while the probes and their respective pre-amplifier boxes (pucks) are separated by $1.5 \mathrm{~m}$. The effective length of the antenna system varies with frequency. For DC and low frequencies (well below the electron plasma frequency $f_{p e}$ ), the effective length is close to the geometric length. For higher frequencies, the effective length is smaller than the physical length, and depends on the frequency in a non-linear way. It may be possible to determine the effective length using whistler mode emissions for calibration (e.g. Hartley et al. 2016).

The red sections in Fig. 4 show the probe and sensor wire, which together form the sensor. The pre-amplifier box ('puck') has two electrically insulated external surfaces, indicated by yellow and blue, the potentials of which can be set individually. The outer puck surface 
Fig. 5 MEFISTO boom unit, interior view (Blomberg et al. 2006)
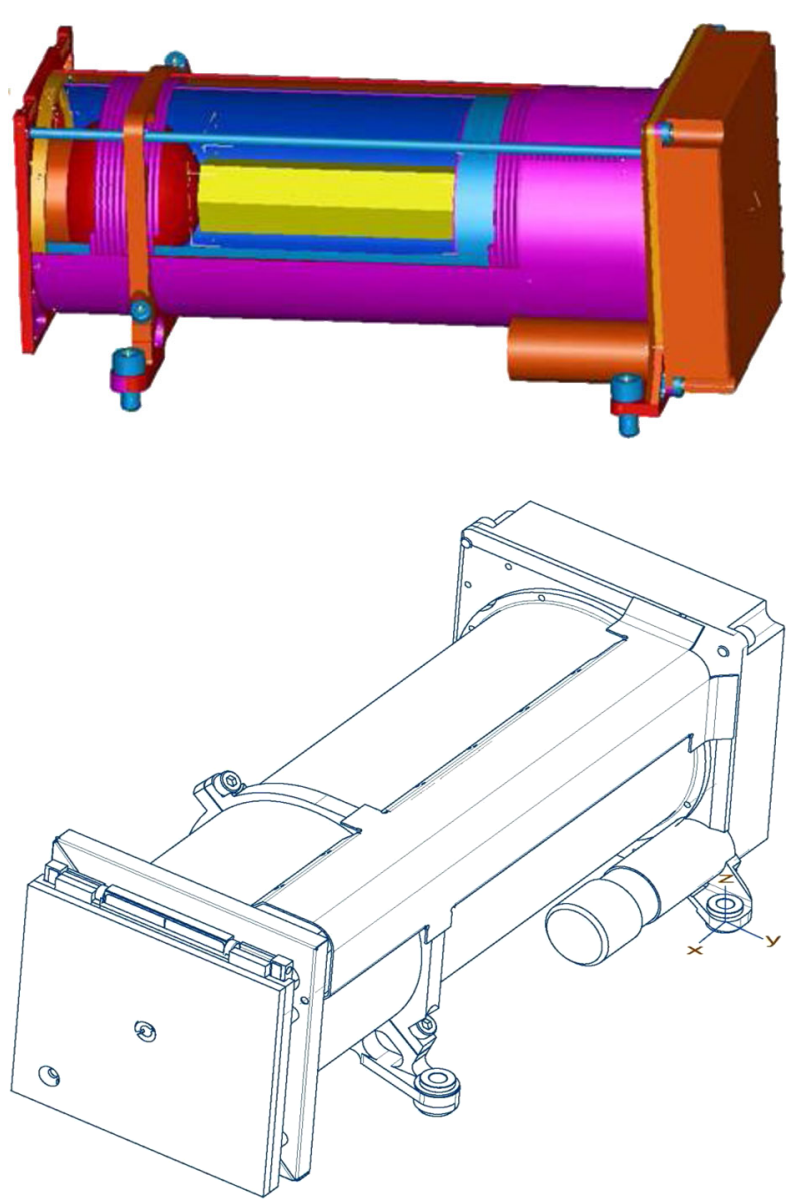

Fig. 6 MEFISTO boom unit, outside view (Blomberg et al. 2006) will probably be kept at a few $\mathrm{V}$ positive with respect to the probe in order to retract photoelectrons emitted by the puck, while the inner puck surface will be kept at about $10 \mathrm{~V}$ negative with respect to the spacecraft body, in order to repel photoelectrons emitted by the boom and by the spacecraft body. The exact values will be decided once MEFISTO has been commissioned in Hermean orbit. The outside of the boom cable is connected to spacecraft ground.

The MEFISTO sensors consist of TiAlN-coated $40 \mathrm{~mm}$ diameter spheres. The sensor is kept as cold as possible in order not to overheat the pre-amplifier electronics in the puck. The electrical properties of the electric field sensors are of crucial importance to the performance of MEFISTO. The homogeneity of the electrical work function to a large degree determines the accuracy of the measurement. The mechanical manufacturing method of the spherical sensor is also an important factor. The electrical work function determines the sensitivity of the surface to EMF effects, which may cause hysteresis in the current-voltage characteristic of the probe when sweeping the bias current.

The boom deployment units (Figs. 5 and 6) are of a new low-mass design and provide storage and deployment of the wire boom, the puck, and the probe. The wire is both a boom and a carrier of the electrical connections between the probes and the experiment unit in the spacecraft body. The boom wire is stored between two concentric cylinders, and is 
fed using a mechanism driven by an electrical motor located at the rear end of the boom unit. The mechanism lifts the wire from its storage between the two cylinders and pushes it out along the central axis. The wire deployment speed is $6.7 \mathrm{~mm} / \mathrm{s}$. The two boom units are mounted on the lower deck, and the probes will be extended through a square hole in the lower spacecraft panel. The front surfaces of the boom units are aligned with the outer surface of the lower panel.

The probe deployment will begin by running a stepper motor; initially running the stepper motor will disengage the lock of the probe housing cover. The probe housing cover is hinged on one side, and opens under spring-loading when released and at about $80^{\circ}$ angle the cover automatically jettisons. The probe release mechanism is located inside the probe and consists of a spiral spring and a reel for the thin (diameter $0.14 \mathrm{~mm}$ ) $1.5 \mathrm{~m}$ long sensor wire. By continuing running the stepper motor the wire boom is deployed. The thin wire mechanism spring force will increase due to the increased centrifugal force and be deployed fully during the first $4.5 \mathrm{~m}$ wire deployment phase. The deployment is done in 3 steps of length $5 \mathrm{~m}, 5 \mathrm{~m}$, and finally $3.3 \mathrm{~m}$ (see also Sect. 4.2).

From a thermal perspective, MEFISTO-S can be divided into five main items: the probe, the thin sensor wire, the pre-amplifier housing, the wire boom, and the boom unit. The constraint on the pre-amplifier housing is to keep the pre-amplifier within a reasonable temperature range, which is taken to be -55 to $125^{\circ} \mathrm{C}$. To increase reliability and reduce current leakage it is preferable to have it below $70{ }^{\circ} \mathrm{C}$. Since the thin sensor wire is made of a Titanium alloy, having a rather low thermal conductivity $(\sim 16 \mathrm{~W} / \mathrm{m} / \mathrm{K})$, the pre-amplifier temperature is mainly set by its surface properties and the ratio between illuminated and radiating area. The pre-amplifier surface is covered with Optical Solar Reflector (OSR) mirrors from Thales, UK, and mounted with a Silicone adhesive. The major constraint on the wire boom is to keep it below $200{ }^{\circ} \mathrm{C}$, which is the upper temperature limit set by the wire insulation material.

\subsection{MEFISTO-E}

The MEFISTO analogue and digital circuitry (see Fig. 7) mounted on one circuit board in the PME chassis, and in the two MEFISTO boom units consists of:

a. two identical pre-amplifiers mounted in each puck, connected to the main electronics board through two $15 \mathrm{~m}$ long nine-wire boom cables

b. two sets of analogue circuitry for controlling the current generator

c. four drivers for controlling the potential of the surfaces on the pucks

d. a common floating ground driver for both probes

e. buffers for distribution of differential and single ended probe DC and HF voltages

f. pulse transformer level shifters for control of floating ground current generators and gain multipliers

g. a Field Programmable Gate Arrays (FPGA) RTAX 1000 controller providing the specialized digital interface to the PWI, motor control, and analogue electronics

h. a dedicated DC/DC converter for handling of floating voltage and floating voltage circuitry power supply

i. power provider, $\pm 100 \mathrm{~V}$ to $\mathrm{EWO}$

j. two motor control units

k. a housekeeping delta sigma ADC.

1. telecommand and housekeeping with DPU with SpaceWire using RMAP protocol @ 2 Mbit/s. 


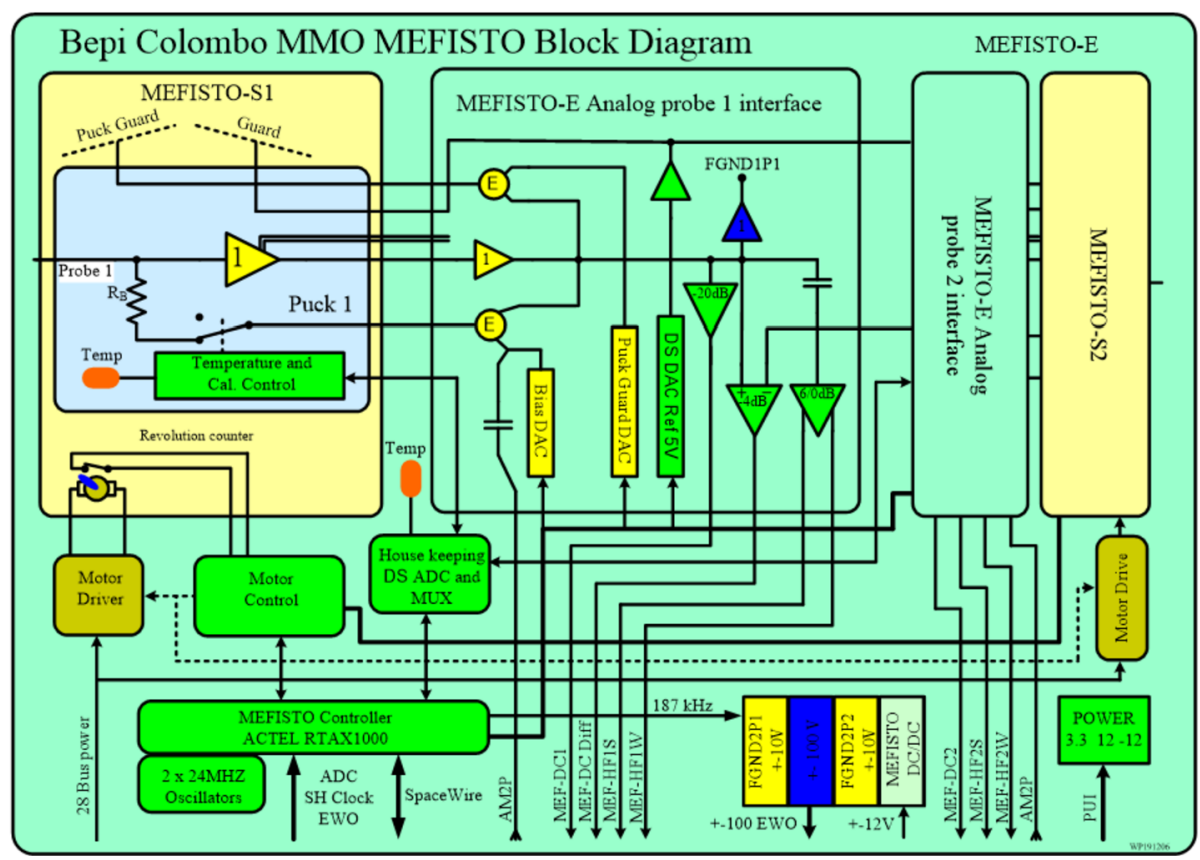

Fig. 7 MEFISTO block diagram

The E-field amplifier circuitry has to deal with input signals far in excess of the input ranges of available components. The solution is to apply a 'floating ground' that follows the signal. While supply voltages are normally referenced to a common ground, the supplies are referenced to this 'floating' ground, which is actually not floating, but connected to a buffered voltage that follows the signal. In the MEFISTO electronics the principle of floating ground is applied as follows: a low-pass filtered value of the probe input potential is used as a floating ground for the pre-amplifier, bias circuitry and stub control. This floating ground has a range of $\pm 100 \mathrm{~V}$ relative to the spacecraft structure. The purpose is to negate the influence of the spacecraft potential, and hold the signal conditioning circuitry near the plasma potential. This also provides a means to monitor the spacecraft potential. Figure 8 illustrates the floating ground concept. The block diagram in Fig. 9 shows the available analog signals provided to $\mathrm{AM}^{2} \mathrm{P}$, EWO and SORBET. It also shows the functionality of the relays controlled by MEFISTO. A1 and $\mathrm{A} 2$ will allow the $\mathrm{AM}^{2} \mathrm{P}$ transmission signal to be output to the two LP probes to enable both self impedance and mutual impedance measurements of the MEFISTO antenna. The B relay is used to ground the boom shield locally at the MEFISTO PCB or to allow $\mathrm{AM}^{2} \mathrm{P}$ to measure the current in the MEFISTO wire boom shield through the $\mathrm{AM}^{2} \mathrm{P}-\mathrm{S}$ current sensor (Fig. 12). The $\mathrm{C} 1$ and $\mathrm{C} 2$ relays controls are used to either completely float the input of the LP amplifier (high impedance mode) or to source a current to the plasma. The D relay allows the E-field signal to EWO and SORBET to have a gain of 0 or $6 \mathrm{~dB}(1 \times$ or $2 \times$ the amplitude).

Each input amplifier measures voltage and provides bias current, using a high input impedance follower with a bootstrapped bias resistor and voltage source. The input stage needs to handle a high source impedance $R$ of $10^{6}-10^{11} \Omega$, and a stray capacitance of $C=14 \mathrm{pF}$. Thus, it must have a low leakage current, $<4 \mathrm{pA}$, for the whole mission, and a 


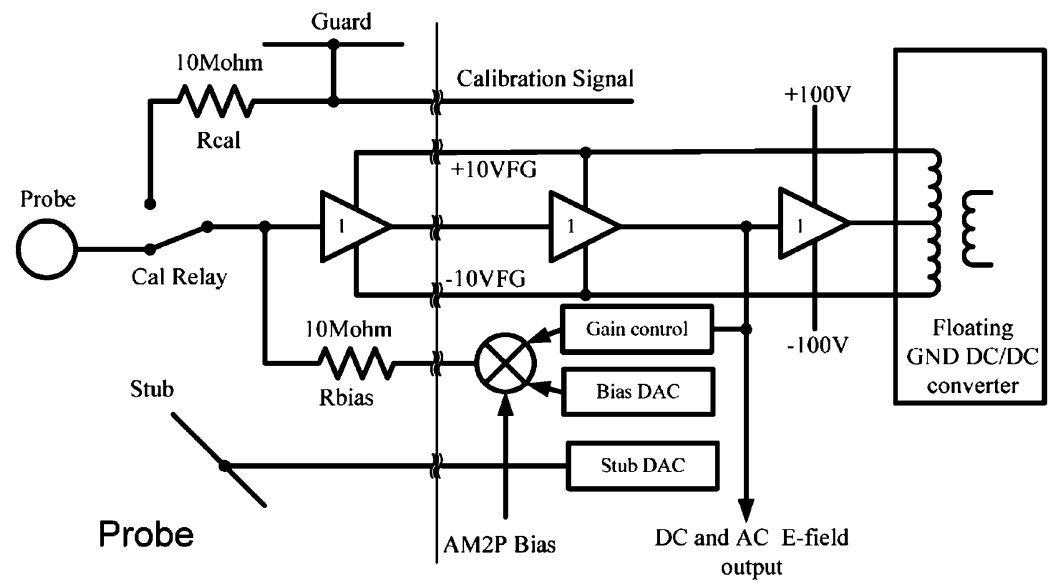

Fig. 8 MEFISTO floating ground concept

\section{MEFISTO Relay Configuration}

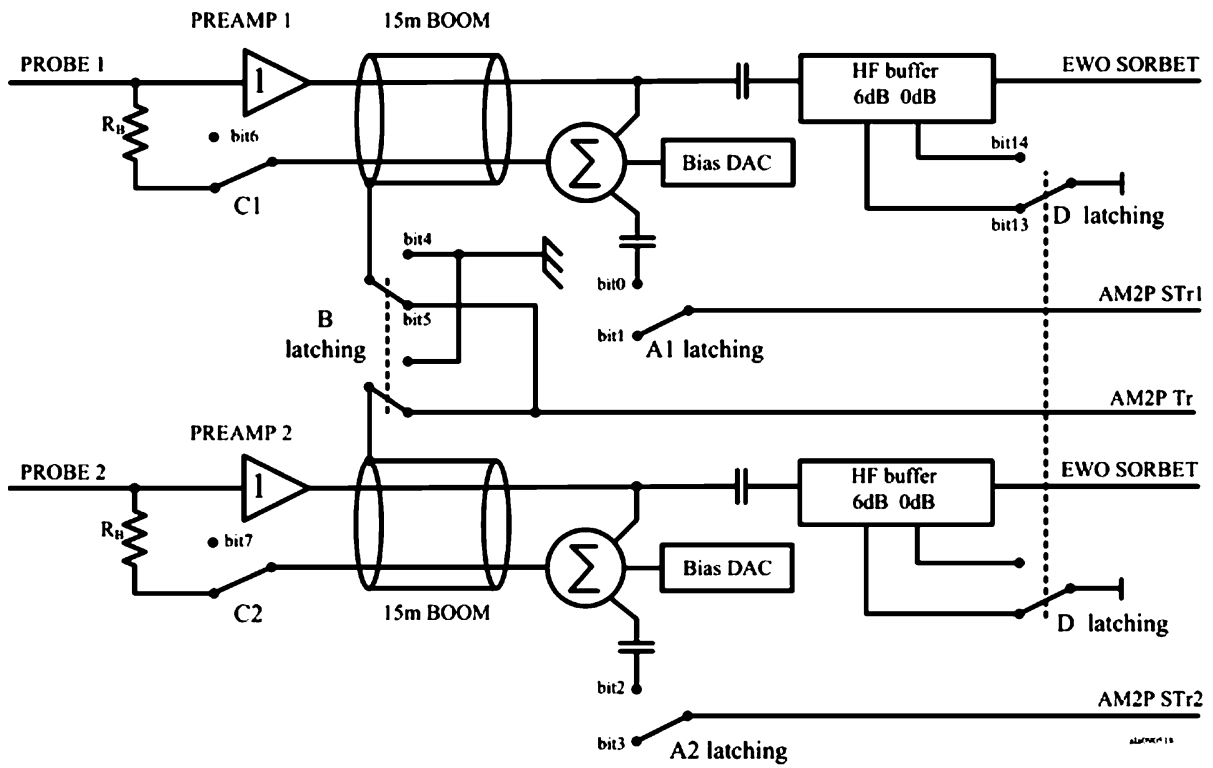

Fig. 9 Illustration of the simplified MEFISTO signal paths and controls

low input capacitance, $<4 \mathrm{pF}$, to minimize attenuation of the input signal. This can only be achieved by proper bootstrapping of the current generator, and by using FET operational amplifiers with low input current, such as the OPA602. It has a radiation tolerance of $>10^{3} \mathrm{~Gy}$, well in line with BepiColombo requirements. The probe potential is determined by the balance of plasma current $\left(i_{\text {plasma }}\right)$ and a bias current $\left(i_{\text {bias }}\right)$ controlled by a current generator. The bias current is produced by controlling the potential across a positive feedback resistor in a voltage follower. The bias current setting is under software control and ranges from 


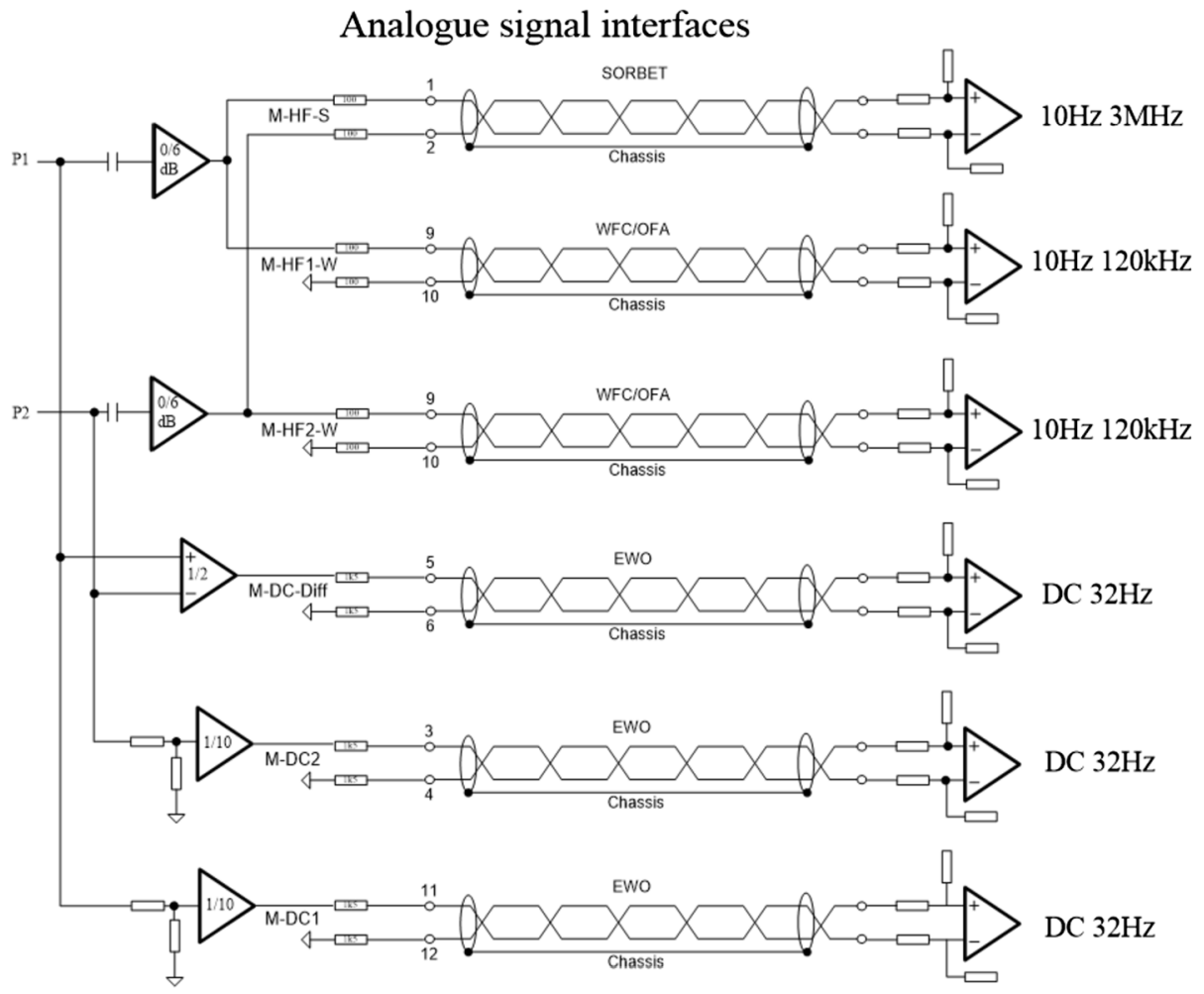

Fig. 10 MEFISTO analogue interfaces

$\pm 3 \mu \mathrm{A}$, with 65536 steps. Each of the stub and guard offset voltages for each of the two probes has a range of $\pm 10 \mathrm{~V}$ and are controlled with 65536 steps.

At regular intervals MEFISTO will perform a current sweep, normally including 64 steps, in order to obtain absolute plasma parameter values from the resulting Langmuir curve. The sweep is controlled by the MDP through the space wire interface and synchronized by the EWO ADC $1024 \mathrm{~Hz}$ sampling clock. During the sweep EWO collects and sends resulting data to MDP for transmission to Earth.

From time to time, the $\mathrm{AM}^{2} \mathrm{P}$ experiment will use the MEFISTO probes to inject an $\mathrm{AC}$ current or voltage, stepped in frequency from 0.7 to $143 \mathrm{kHz}$, into the plasma by modulating the MEFISTO current generator, in order to determine the self and mutual impedance of the MEFISTO antenna immersed in the plasma to be diagnosed. The signal response (voltage or current) is simultaneously measured and analyzed in the EWO-WFC/OFA receiver. In the $\mathrm{AM}^{2} \mathrm{P}$ double wire mode and mutual mode the MEFISTO wire boom shields are used as transmitters and the corresponding shield currents are measured by the current probe $\mathrm{AM}^{2} \mathrm{P}-\mathrm{S}$ hosted in each of the MEFISTO deployment boom units (Fig. 12). When not used by $\mathrm{AM}^{2} \mathrm{P}$, the boom wire shields are connected via a relay or a multiplexer to ground. $\mathrm{AM}^{2} \mathrm{P}$ also provides $\mathrm{AC}$ current injections in the frequency range $2.6 \mathrm{~Hz}$ to a few $\mathrm{kHz}$ for the MEFISTO - WPT probe impedance cross-calibration. The analog interfaces to the other instruments is illustrated in Fig. 10.

The MEFISTO boom deployment is controlled by a stepper motor. The step signals are generated by a digital controller executed in the MEFISTO-E FPGA. The deployment is 
programmable in orbit as is the deployment sequence and speed profile. The control signals are transferred to the motor driver via an isolated interface to meet the ground separation requirement. The boom deployment is monitored by counting motor steps, and verified by a micro switch, counting turns on a wheel of the mechanism.

\subsection{Science Objectives}

Since the MEFISTO instrument is partially designed to deliver high-quality low-frequency electric field data, we here discuss some scientific objectives that are associated with DC and low-frequency (LF) electric fields. Further scientific goals can be found in the PWI overview paper (Kasaba et al. 2020).

\subsubsection{Magnetosphere}

Plasma Convection The electric field can provide the plasma $\mathbf{E} \times \mathbf{B}$ flow in the magnetosphere on DC to LF time scales, and provide an important complement to the flow determined by ion moment calculations. This includes the large-scale magnetospheric convection, as well as possible localized bursty bulk flow-like structures (Dewey et al. 2018). Statistically, also the large-scale solar wind-magnetosphere interaction can be studied by measurements of the total polar cap electric potential drop (e.g. Blomberg et al. 2006). Deviations between particle motion and $\mathbf{E} \times \mathbf{B}$ drift can also indicate regions where the plasma no longer exhibits frozen-in behaviour, indicating ongoing reconnection (see below).

Acceleration Potential Structures While Mercury only has a very thin exosphere, and therefore a very weak ionosphere (e.g. Wurz et al. 2019), field-aligned currents from the magnetotail seem to close in the vicinity of the planet, possibly in the planetary core (Anderson et al. 2014). When these field-aligned currents are large, field-aligned electric fields may be set up to ensure current continuity, in analogy with processes in Earth's magnetosphere. Such acceleration structures also give rise to quasi-static perpendicular electric fields (e.g. Karlsson 2012, and references therein), which may be measured by MEFISTO.

Substorms The largest field-aligned currents will probably be associated with substorm dipolarizations, that have been observed in the Mercury magnetosphere (Sun et al. 2015), which may trigger unusually large acceleration potentials. Substorm injections are also likely to produced induction electric fields (e.g. Gabrielse et al. 2012). Studying these with MEFISTO will increase our understanding of injection of energetic particles in the inner magnetosphere (Sun et al. 2017).

Reconnection The Mercury magnetosphere is strongly driven by reconnection, taking place both on the day side and in the magnetotail, often taking the form of flux transfer events (Slavin et al. 2010, 2012, 2014, 2019; Imber and Slavin 2017). In studying reconnection at Mercury, electric field observations will provide new information (e.g. Lindqvist et al. 2016). The electric field parallel to reconnection currents sheets will, for example, give direct information on the reconnection rate, while electric fields parallel to the magnetic field are responsible for particle acceleration. Low-frequency waves may play an important role in the onset of reconnection, and the electric field observations will be important in identifying wave modes. 
Alfvén Waves and Field-Aligned Resonances Ultra-low frequency waves in the range of $\mathrm{mHz}$ to around $1 \mathrm{~Hz}$ have been extensively observed inside the Hermean magnetosphere (Russell 1989; James et al. 2016; Boardsen et al. 2012; Liljeblad et al. 2016; Liljeblad and Karlsson 2017). However, the nature of these waves is still unclear, in particular their propagation properties, or the nature of their reflection at the exosphere or planetary surface. Electric field measurements will provide phase differences between electric and magnetic wave components, which will greatly help in resolving these questions. Studying reflective properties as a function of frequency will also give information on the conductivities of the reflective media.

\subsubsection{Solar Wind and Magnetosheath}

Low-frequency measurements in the solar wind and magnetosheath will also be of great use in addressing a number of science questions.

Pickup Ions Recent Rosetta observations from comet 67P show that pickup ions with gyro radii comparable to the scale of the object interacting with the solar wind may set up polarization electric fields (e.g. Behar et al. 2016). Such fields can strongly effect the particle dynamics around the planet, and the re-population of the magnetosphere and will be investigated.

Foreshock Waves and Structures When the solar wind magnetic field is close to parallel to the bow shock normal, a foreshock with reflected solar wind particles is formed. This region is rich in wave phenomena in the linear and possibly non-linear phases (Le et al. 2013; Jarvinen et al. 2019), which may also effect the properties of the magnetosheath and ultimately interact with the magnetopause (Sundberg et al. 2013). As in most cases when studying waves, the electric field will provide important information on their properties and generation.

Magnetic Holes A recent finding is that small-scale irregularities in the solar wind, magnetic holes, with scales smaller than the Mercury magnetosphere will probably penetrate the bow shock, and interact with the magnetopause (Karlsson et al. 2016). The electric field will be an important indicator of how this interaction can trigger local reconnection or ULF wave activity.

Solar Wind - Magnetosphere Interactions at Low Latitude BepiColombo will enable investigations of the nature of the interaction between the solar wind and the magnetosphere of Mercury. Among other processes, velocity shear instabilities such as the KelvinHelmholtz instability (KHI) shall be observed though its LF electric field signatures. This large scale instability has been observed to be the driver of secondary instabilities at smaller, kinetic scales, both in Earth observations ( $\mathrm{Li}$ et al. 2016) and in kinetic numerical simulations (Henri et al. 2013), and can significantly enhance energy and particle transport between the solar wind and the magnetosphere. One of the surprises from the MESSENGER mission is however that the velocity shear layer between the magnetosheath and the magnetosphere of Mercury seems to be unstable to the KHI only on the dusk side of the magnetosphere (Sundberg et al. 2012). In this context, other instabilities, that can build on the free energy available at the magnetopause and compete with the KHI in an asymmetric velocity shear, shall leave a significant electric field fingerprints to be observed by MEFISTO. This is for instance the case of the lower-hybrid drift instability that might build on the strong density gradients at the Hermean magnetopause and reduce or even suppress the development of the KHI (Dargent et al. 2019). 
Dust Interplanetary dust inside Earth's orbit has been observed with the zodiacal light and the solar F-corona (e.g. Mann 2017). Some in situ measurements of dust grains with size down to 1 micrometer have been provided by the Helios missions (Altobelli et al. 2006). Recent in-situ observations suggest an outgoing flux of the nanograins near Earth's orbit (Meyer-Vernet et al. 2009). The presence of a circumsolar ring near Mercury's orbit was recently recognised as well (Stenborg et al. 2018). Dust affects the surface and the exosphere of the Mercury. Micrometeorites bombardment causes space weathering and impact vaporization alter the composition of the exosphere (e.g. Syal et al. 2015; Gamborino et al. 2019).

The PWI antennas can be used to detect sub-micrometer to micrometer-sized dust, called $\beta$ particles, in situ. A dust particle impact to either the spacecraft body or the electric field antenna produces a plasma cloud around the spacecraft which will be detected by the onboard electric antennas. This technique has been used by the many other spacecraft, e.g., Voyagers, Cassini, and STEREO (e.g. Zaslavsky et al. 2012). The BepiColombo MMO also carries a dust detector (MDM) for the first-time near Mercury orbit. Comparing the independently obtained dust measurements will provide detailed characteristics of the dust environment around at Mercury. $\beta$ particles are affected by the gravitational force as well as the radiation and Lorentz forces, which determines their dynamics. When the grains are immersed in a plasma, they acquire charges depending on the surrounding plasma environment. Consequently, the solar wind properties affect the dynamics of the grains. The MMO/PWI measures the electrical potential of the spacecraft which, in turn, gives information of the electrical potential of the dust grains which is a key parameter to define the dust dynamics.

\section{WPT-S}

The WPT system comprises a pair of wire boom sensors. Each boom consists of the wire with the length of $15 \mathrm{~m}$ with a sphere at the ends. Both booms are deployed to opposite direction parallel to the spin plane.

The design is based on the heritage of the Geotail PANT system, which was connected to two receivers: the Electric Field Detector (EFD) for lower frequencies (Tsuruda et al. 1994) and the Plasma Wave Instrument (PWI) for higher frequencies up to $800 \mathrm{kHz}$ (Matsumoto et al. 1994). Also the JAXA Arase spacecraft used almost identical antennas, the PWE WPT system (Kasahara et al. 2018; Kasaba et al. 2017). From its launch in late 2016, the Arase WPT system worked well. The performance of the Arase WPT system in Earth orbit was summarized in Kasaba et al. (2017).

Figure 11 shows a schematic drawing of WPT-S after the deployment. The deployment units containing motors are installed at the outer side of the spacecraft bottom panel and just inside of the spacecraft side panel. In this configuration, the wire root position is at the distance of $0.8 \mathrm{~m}$ from the spacecraft spin axis. After the full deployment, a pair of WPT wire booms form a dipole antenna with $31.6 \mathrm{~m}$ tip-to-tip length.

Each wire antenna boom is composed of a conductive wire with the length of $15 \mathrm{~m}$ and a conductive sphere at the end with a diameter of $60 \mathrm{~mm}$. The wire has a conductive core made of stainless steel (SUS316L, diameter: $0.18 \mathrm{~mm}$ ) covered by a polyimide insulator (diameter: $0.43 \mathrm{~mm}$ ), except for a region at the end of the wire with a length of $0.7 \mathrm{~m}$. The sphere is a Ti-alloy (TI-6AL-4V) shell with thickness of less than $1 \mathrm{~mm}$. In this design, the conductive end part, i.e., the sphere (60 $\mathrm{mm}$ in diameter) and the conductive wire without polyimide cover $(0.7 \mathrm{~m}$ in length, with the cross section less than $5 \%$ of the sphere's), forms the probe which is exposed to the ambient plasma, while the remaining wire $(14.3 \mathrm{~m}$ in 


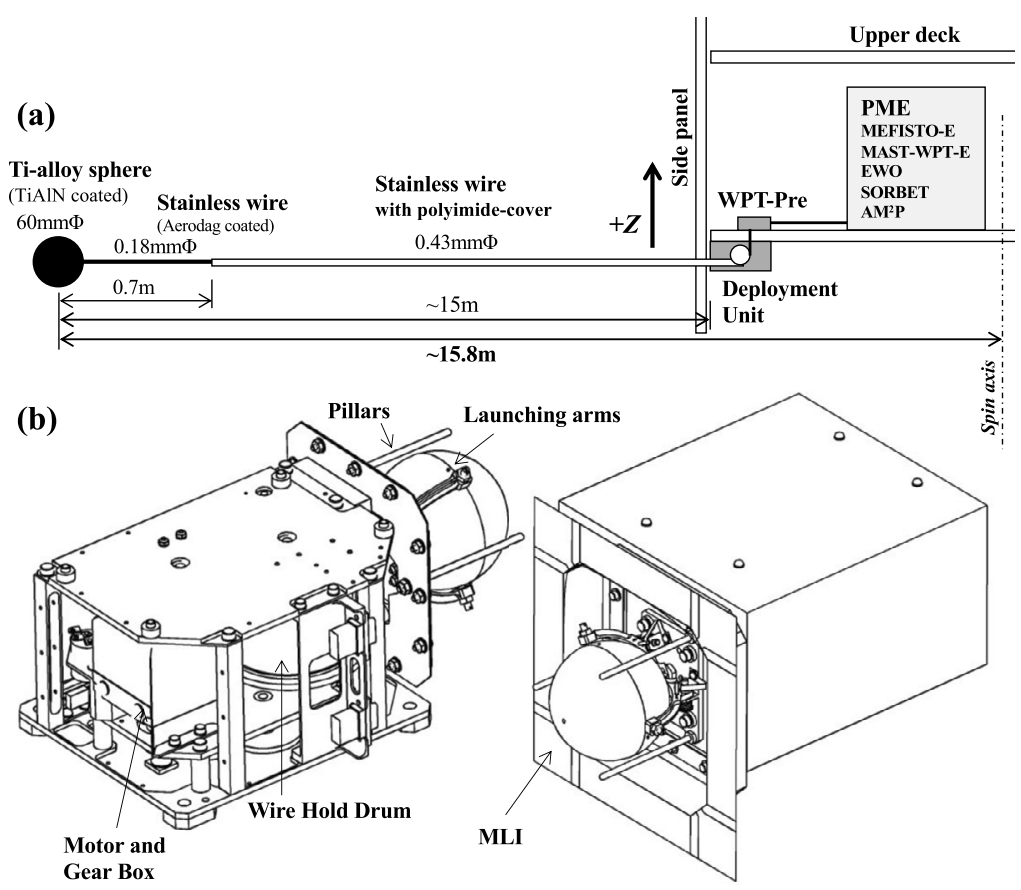

Fig. 11 Design of WPT-S. (a) Geometry. (b) Schematic drawing. To the left the mechanics of the deployment unit is shown. To the right is the outer view covered by MLI. (b) is courtesy of Nippi Corporation

length) is insulated from the plasma. This design is similar to Arase PWE WPT-S except for the material of the sphere. For Mio, in order to survive the hotter environment, the Al alloy which was used in Arase was replaced by a Ti alloy.

For stable potential measurement of the ambient plasma, the surface of the conductive part is requested to have uniform conductivity, photoelectron emissivity, and secondary photoelectron yield. The sphere surface is coated with TiAlN with a Ti:Al ratio of 2:1 and a thickness of $1 \mu \mathrm{m}$. The outer, exposed conductive part of the wire is coated by Aerodag (a graphite paint). This part is not only there for facilitating the contact to the ambient plasma, but also for reducing the influence from the floating potential of the insulated surface on the long wire boom to the sphere at the end. The cross section of this region is required to be as small as possible, in order to reduce the variation of photoelectron emission from the conductive wire part modulated by spacecraft spin rotation. The remaining polyimide region with a length of $14.3 \mathrm{~m}$ isolates the resistive coupling of the long wire core to the ambient plasma. Inside of the deployment unit, the wire is covered with a copper-meshed shield (diameter: $0.61 \mathrm{~mm}$ ) for prevention of a capacity coupling between the sensitive wire part to the deployment unit which is grounded to spacecraft body.

In the DC and low-frequency range (less than several tens of $\mathrm{Hz}$ ) this design ensures that WPT-S couples to the surrounding plasma only at its end conductive part. In this case, the effective antenna length in this frequency range is expected to be similar to the tip-to-tip length, $31.6 \mathrm{~m}$. By a suitable bias current fed from PWI EWO-EFD, an effective coupling resistance to ambient plasma is expected to be a few to a few tens of $\mathrm{M} \Omega$. In orbit around Mercury, the photoelectron current from the WPT-S sphere and exposed conductive wire is expected to vary with solar distance (0.31-0.47 AU) and will be about $250-1,500 \mathrm{nA}$. The 
Fig. 12 Photo of the $\mathrm{AM}^{2} \mathrm{P}-\mathrm{S}$ current probe, hosted in the MEFISTO deployment box, designed to measure the current in the MEFISTO wire boom shield

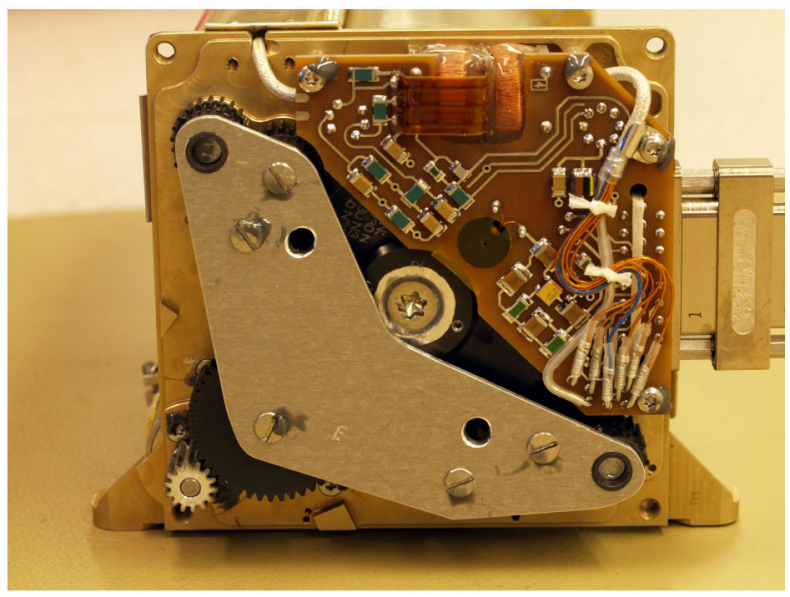

EWO-EFD is designed to feed the bias current with a value of $-1,500 \mathrm{nA}$ to $+1,500 \mathrm{nA}$ in 256 steps.

In the higher frequency range, WPT-S acts as a wire antenna of $31.6 \mathrm{~m}$ with a capacitive coupling to the ambient plasma. For each monopole antenna, the capacitance of the long wire part $C_{\text {wire }}$ and sphere part $C_{\text {sphere }}$ in vacuum are expected to be 35 and $3.4 \mathrm{pF}$, respectively. In real space, plasma sheath impedance also needs to be taken into account. For the Arase WPT in Earth orbit, the measured coupling capacitance in the dipole antenna configuration was $75-85 \mathrm{pF}$, and the effective antenna length about half the geometric length, $16 \mathrm{~m}$.

\subsection{Deployment Unit of WPT-S}

The deployment unit of WPT-S consists of two parts; the motor box and the sphere holder. As seen in Fig. 11, the motor box is installed at the edge and on the outer side of the lower deck, just behind the side panel which acts as sun shield for the instrument inside of the spacecraft. It contains a wire hold drum and a stepping motor with a gear box. The antenna wire is stowed on the wire hold drum, and is electrically connected to an output signal line by a slip ring. The output signal line is connected to the input of WPT-Pre located on the inner side of the lower deck.

The sphere holder on the motor box is located on the outer side of the spacecraft side panel through a square hole, and will be exposed to strong sunlight in Mercury orbit. It holds the sphere sensor with three latching arms. This is a launch lock system and can be opened by a small rotation of the motor drive. It was released in the early interplanetary cruise phase (see Sect. 4.1), in order to reduce risky mechanical actions in high temperature under high solar illumination. After its release, the sphere can be supported by the balance of the sensor wire (pulling force) and the spring pusher (pushing force). Three pillars around the sphere act as a backup guard for free motion of the sphere.

In order to reduce the heat flow into the spacecraft as much as possible, the sphere holder is thermally insulated from the motor box (and the spacecraft lower deck). The base of the sphere holder is made of polyimide for thermal insulation. The motor box is also insulated from the spacecraft lower deck by similar way. In addition, the gaps between the deployment unit and the side panel are covered by Multi-Layer Insulator (MLI). This design has been well checked and validated by careful analysis and prelaunch tests. 


\subsection{WPT Preamplifiers}

The output signal from WPT-S is fed to EWO and SORBET through WPT-Pre, which are identical pre-amplifiers connected to each of the two WPT-S wires. They cover a wide frequency range from DC to $10 \mathrm{MHz}$. Because it is not a floating-type amplifier, WPT-Pre contains two amplifiers connected to WPT-S in parallel, WPT-Pre(DC) and WPT-Pre(AC).

WPT-Pre(DC) covers a wider dynamic range, $+/-100 \mathrm{~V}$ for EWO-EFD, measuring the signal at frequencies from DC to $32 \mathrm{~Hz}$. It is connected to the WPT-S through a voltagedivider circuit (10 G $\Omega$ and $1 \mathrm{G} \Omega$ ) and has a gain of $-20 \mathrm{~dB}$. It also has a bias current feed function, which can also be used for resistive impedance measurement of the WPT-S antenna to ambient plasmas.

WPT-Pre(AC) covers the frequency range from $10 \mathrm{~Hz}$ to $10 \mathrm{MHz}$ for EWO-OFA/WFC and SORBET. Its input is decoupled from large input potentials by a capacitance $(200 \mathrm{pF})$. It has a gain of $20 \mathrm{~dB}$ in order to realize the lower noise floor, and also has an attenuator of $-20 \mathrm{~dB}$ in order to avoid possible saturation. WPT-Pre(AC) has a calibration signal feed line, whose drive pattern is provided from the $\mathrm{AM}^{2} \mathrm{P}$ synthesizer. It can be used for capacitive impedance measurement of the WPT-S antenna to the ambient plasma.

Detailed electrical functionality and performance of WPT-Pre is described in Kasaba et al. (2020). A similar design was used for the Arase WPT preamplifiers (Kasahara et al. 2018; Kasaba et al. 2020) and showed good results. We will expect a good performance at Mercury.

\section{Commissioning Operation and Deployment of Wire Antennas}

Commissioning operations of PWI, including the MEFISTO and WPT systems, were successfully executed in November 2018, one month after the launch. All functions and performance in the stowed configuration were checked, and we successfully confirmed the health of the electric field sensor system. During the long interplanetary cruise phase, both antennas will be behind the BepiColombo MOSIF (Magnetospheric Orbiter Sunshield and Interface Structure) and guarded from intense sun light. Unfortunately, the WPT-S sensors are electrically grounded to the spacecraft with low impedance and have no observational capability. The MEFISTO-S sensors are floating, but are located inside an aluminum cover, and also have no observation capability. Observations will start from 2026 in Mercury orbit after the most critical event, full deployment of MEFISTO-S and WPT-S.

\subsection{Latch Release of WPT-S During Near Earth Commissioning Phase}

The WPT-S deployment unit has a sphere holder, which consists of three latching arms as a launch lock and holds the sensor sphere at the end of the long conductive wire (Fig. 11b). A small motor rotation can move these arms slightly, and open this launch lock. This action was planned before the arrival to Mercury when the Mio spacecraft was guarded by MOSIF, in order to avoid critical mechanical actions under harsh solar illumination.

This action succeeded on 7 August 2019. Figure 13 shows the SORBET frequency-time diagram which shows the dipole signal from the WPT-S1 and WPT-S2 pair before and after the latch release actions. At the latch release of WPT-S1 (10:45 UT), the noise floor in several $\mathrm{kHz}$ - several $100 \mathrm{kHz}$ are clearly reduced. At the latch release of WPT-S2 (11:58 UT), the noise floor in the range of several $\mathrm{kHz}$ to several $100 \mathrm{kHz}$ is slightly increased. The interpretation is that the conductive or radiative noise picked up by the WPT-S1 and WPT-S2 is affected by the impedance change between WPT-S1 - Spacecraft and WPT-S2 - Spacecraft. 


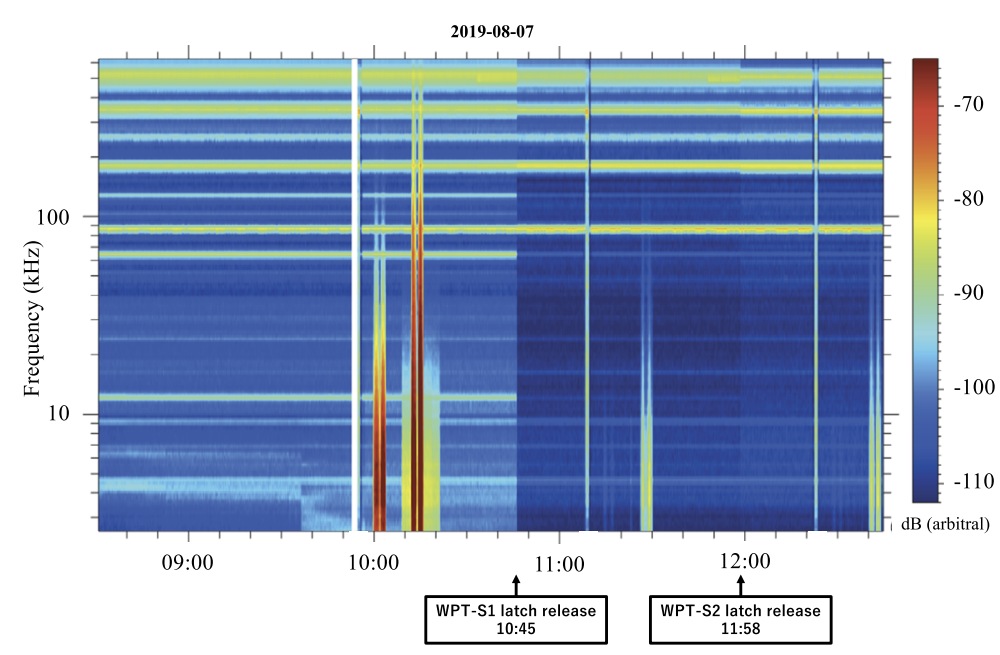

Fig. 13 Noise floor changes seen in PWI SORBET spectrum during the WPT-S latch release operations on 7 August 2019. The latch release action of WPT-S1 and WPT-S2 were at 10:45 UT and 11:58 UT, respectively

We have also checked the resistive impedance between WPT-S1 - Spacecraft and WPT$\mathrm{S} 2$ - Spacecraft by the response to the bias current fed from EWO-EFD to WPT-Preamp. Before the latch release actions, the connection of the WPT-S probes to the spacecraft body through the latching arms was not electrically stable. The measured impedance was about $100-500 \mathrm{k} \Omega$ for WPT-S1 and $1 \mathrm{k} \Omega$ for WPT-S2. After the latch release actions, both spheres were pushed by the pusher spring. In this configuration, similar resistances of about $4-5 \mathrm{k} \Omega$ were detected. Those values agree with the planned values and the ones measured on the engineering model on the ground. These results also assured the electrical health of WPT-S, WPT-Pre, EWO, and SORBET. The sphere and wires of the WPT-S were well connected from the root and the end, and the functions and performance of amplifiers and receivers showed no problems.

\subsection{Deployment of Wire Antennas in Mercury Orbit}

The full deployment of MEFISTO-S and WPT-S booms will be performed in early 2026, after Mercury orbit insertion and separation of the Mio spacecraft from the BepiColombo cruise composite. The deployment action will take place under intense solar illumination. These operations will be performed under the responsibility of the Mio system Team. Since it will be executed at a location far from the Earth, real time monitors and controls will be impossible. The operation will be controlled automatically with safety measures and backup scenarios. It will be the first wire antenna deployment in planetary orbit. (It was planned for the Nozomi spacecraft to Mars (Matsumoto et al. 1998), but not executed because of system failures.)

The basic plan of the deployment operations is as follows: (1) Spin up (spin rate: increased from 15 to $24 \mathrm{rpm}$ ). (2) WPT-S and MEFISTO-S deployment (spin rate: decreased to $11 \mathrm{rpm}$ ). (3) MAST-MGF and MAST-SC deployment (spin rate: decreased to $7 \mathrm{rpm}$ ), and (4) Spin up to the normal operation value (spin rate: increased to $15 \mathrm{rpm}$ ). Wire antenna deployment will be done before the MAST deployment, similar to the deployment operations of Geotail, Nozomi, and Arase (e.g. Kasaba et al. 2017). This order is because (a) the wire 
antennas require higher spin rate at the initial deployment in order to obtain sufficient centrifugal force which can pull out the wire from the deployment unit, and (b) MAST requires a lower spin rate in its deployment phase.

In order to retain dynamic balance of the spacecraft, the deployment operation of MEFISTO and WPT will preferably be executed simultaneously. However, due to limitations of the capability of the PME power supply, simultaneous motor drive of both MEFISTO and WPT is not possible. Therefore, the MEFISTO-S pair and the WPT-S pair are alternatively deployed with 3 steps, i.e., from $0 \mathrm{~m}$ to $5 \mathrm{~m}$, from $5 \mathrm{~m}$ to $10 \mathrm{~m}$, and from $10 \mathrm{~m}$ to full extension $(15 \mathrm{~m})$.

After the deployment, the stable photoelectron emissions from the sphere and the conductive part of the wire are essential for the stability of probe potentials and good electric field measurements. This requires minimizing the shadow of the spacecraft on the wire antenna. For this purpose, the spin axis of the Mio spacecraft will be inclined at 87-88 degrees relative to the ecliptic plane. In this case, the length of the shadow casting on the wire booms by the side panel (extended to $15 \mathrm{~cm}$ below the lower deck) is about $3 \mathrm{~m}$. Although the shadow of the Middle Gain Antenna (MGA; extended $70 \mathrm{~cm}$ below the lower deck) is about $15 \mathrm{~m}$, this effect is not critical because its shadow close to the sphere can be treated as a penumbra, i.e., the angular size of Sun is larger than the angular width of the MGA in the view from the end of the wire antennas.

This requirement increases the spacecraft thermal potential, because the sunlight can partially illuminate the inner side of the side panel below the lower deck, which acts as a radiator in the system design. In the hot case near the periapsis, the spin axis will need to be kept perpendicular to the Sun to avoid the illumination of the lower deck, since spacecraft safety is always the top priority. In this case, the end of the wire antennas will be affected by the spacecraft shadow and we will need to take care of noise pulses in the probe potentials caused by the shadows on the spheres.

\section{Summary}

In the present paper, we have introduced specifications and cruise status of the MEFISTO and WPT systems of the PWI on the BepiColombo Mio spacecraft. The functions and performances were well tested with the PWI system during the Mio system test campaign at JAXA ISAS until 2015, the BepiColombo system test campaign at ESA ESTEC, and the launch campaign at Kourou. Now, we are awaiting the full deployment in early 2026, after the BepiColombo arrival to Mercury. Although the MESSENGER mission provided multiple new topics of Mercury, electric field observations were not performed. Observation from PWI electric field sensors around Mercury will ensure new findings and information on the structure, dynamics, and interactions in this harsh planetary environment.

\footnotetext{
Acknowledgements The authors, the MEFISTO and WPT members of the BepiColombo MMO PWI team, thanks to all members participating to and supporting us. The authors are also grateful to all Mio and BepiColombo project members who are also waiting the successful end of the long journey to this hottest planet. MEFISTO was supported by SNSA (Sweden) and NSC (Norway). WPT was supported by JAXA (Japan). $\mathrm{AM}^{2} \mathrm{P}$ is supported by CNES and CNRS. YK deeply thanks all colleagues of NIPPI Co. Ltd (K. Hamada, J. Kurihara, O. Maeda, M. Makita, K. Okazaki, Y. Ono, T. Sasaki, H. Sato, Y. Takeuchi, T. Yumoto) and S. Shinoda for the designs, developments, and tests of WPT during more than 10 years from the beginning of this project in early 2000 s. TK wants to express his thanks to everyone involved in the development of MEFISTO over the years. This effort is due to the hard work of many people, and we are deeply grateful for those efforts. Finally, the PWI team would like to thank the Mio operation members who assisted the commissioning operations including the latch release actions and will conduct the full deployment operations at Mercury together with us.
} 
Funding Note Open access funding provided by Royal Institute of Technology.

Conflict of interest The authors declare that they have no conflict of interest.

Publisher's Note Springer Nature remains neutral with regard to jurisdictional claims in published maps and institutional affiliations.

Open Access This article is licensed under a Creative Commons Attribution 4.0 International License, which permits use, sharing, adaptation, distribution and reproduction in any medium or format, as long as you give appropriate credit to the original author(s) and the source, provide a link to the Creative Commons licence, and indicate if changes were made. The images or other third party material in this article are included in the article's Creative Commons licence, unless indicated otherwise in a credit line to the material. If material is not included in the article's Creative Commons licence and your intended use is not permitted by statutory regulation or exceeds the permitted use, you will need to obtain permission directly from the copyright holder. To view a copy of this licence, visit http://creativecommons.org/licenses/by/4.0/.

\section{References}

N. Altobelli, E. Grün, M. Landgraf, A new look into the helios dust experiment data: presence of interstellar dust inside the Earth's orbit. Astron. Astrophys. 448(1), 243-252 (2006)

B.J. Anderson, C.L. Johnson, H. Korth, J.A. Slavin, R.M. Winslow, R.J. Phillips, R.L. McNutt, S.C. Solomon, Steady-state field-aligned currents at Mercury. Geophys. Res. Lett. 41(21), 7444-7452 (2014)

E. Behar, H. Nilsson, G.S. Wieser, Z. Nemeth, T. Broiles, I. Richter, Mass loading at 67P/ChuryumovGerasimenko: a case study. Geophys. Res. Lett. 43(4), 1411-1418 (2016)

J. Benkhoff et al., Tbw. Space Sci. Rev. (2020, this issue)

J. Benkhoff, J. van Casteren, H. Hayakawa, M. Fujimoto, H. Laakso, M. Novara, P. Ferri, H. Middleton, R. Ziethe, BepiColombo - comprehensive exploration of Mercury: mission overview and science goals. Planet. Space Sci. 58, 2-20 (2010). https://doi.org/10.1016/j.pss.2009.09.020

L.G. Blomberg, H. Matsumoto, J.L. Bougeret, H. Kojima, S. Yagitani, J.A. Cumnock, A. Eriksson, G.T. Marklund, J.E. Wahlund, L. Bylander et al., MEFISTO - an electric field instrument for BepiColombo/MMO. Adv. Space Res. 38(4), 672-679 (2006). https://doi.org/10.1016/j.asr.2005.05.032

S.A. Boardsen, J.A. Slavin, B.J. Anderson, H. Korth, D. Schriver, S.C. Solomon, Survey of coherent $\sim 1 \mathrm{~Hz}$ waves in Mercury's inner magnetosphere from MESSENGER observations. J. Geophys. Res. Space Phys. 117(A12), A00M05 (2012)

H. Breuillard, P. Henri, L. Bucciantini, M. Volwerk, T. Karlsson, A. Eriksson, F. Johansson, E. Odelstad, I. Richter, C. Goetz, X. Vallières, R. Hajra, Properties of the singing comet waves in the 67P/ChuryumovGerasimenko plasma environment as observed by the Rosetta mission. Astron. Astrophys. 630, A39 (2019). https://doi.org/10.1051/0004-6361/201834876

J. Dargent, F. Lavorenti, F. Califano, P. Henri, F. Pucci, S.S. Cerri, Interplay between Kelvin-Helmholtz and lower-hybrid drift instabilities. J. Plasma Phys. 85(6), 805850601 (2019). https://doi.org/10.1017/ S0022377819000758

R.M. Dewey, J.M. Raines, W. Sun, J.A. Slavin, G. Poh, MESSENGER observations of fast plasma flows in Mercury's magnetotail. Geophys. Res. Lett. 45(19), 10-110 (2018)

R. Ergun, C. Carlson, F. Mozer, G. Delory, M. Temerin, J. McFadden, D. Pankow, R. Abiad, P. Harvey, R. Wilkes et al., The fast satellite fields instrument, in The FAST Mission (Springer, Berlin, 2001), pp. 6791

C. Gabrielse, V. Angelopoulos, A. Runov, D. Turner, The effects of transient, localized electric fields on equatorial electron acceleration and transport toward the inner magnetosphere. J. Geophys. Res. Space Phys. 117(A10), A10213 (2012)

D. Gamborino, A.H. Vorburger, P. Wurz, Mercury's subsolar sodium exosphere: an ab initio calculation to interpret MASCS/UVVS observations from MESSENGER. Ann. Geophys. 37, 455-470 (2019)

G. Gustafsson, M. André, T. Carozzi, A.I. Eriksson, C.G. Fälthammar, R. Grard, G. Holmgren, J. Holtet, N. Ivchenko, T. Karlsson et al., First results of electric field and density observations by Cluster EFW based on initial months of operation. Ann. Geophys. 19, 1219-1240 (2001)

D. Hartley, C. Kletzing, W. Kurth, S. Bounds, T. Averkamp, G. Hospodarsky, J.R. Wygant, J. Bonnell, O. Santolík, C.E. Watt, Using the cold plasma dispersion relation and whistler mode waves to quantify the antenna sheath impedance of the Van Allen Probes EFW instrument. J. Geophys. Res. Space Phys. 121(5), 4590-4606 (2016) 
H. Hayakawa, Y. Kasaba, H. Yamakawa, H. Ogawa, T. Mukai, The BepiColombo/MMO model payload and operation plan. Adv. Space Res. 33(12), 2142-2146 (2004). https://doi.org/10.1016/ s0273-1177(03)00438-1

P. Henri, S.S. Cerri, F. Califano, F. Pegoraro, C. Rossi, M. Faganello, O. Šebek, P.M. Trávníček, P. Hellinger, J.T. Frederiksen, A. Nordlund, S. Markidis, R. Keppens, G. Lapenta, Nonlinear evolution of the magnetized Kelvin-Helmholtz instability: from fluid to kinetic modeling. Phys. Plasmas 20(10), 102118 (2013). https://doi.org/10.1063/1.4826214

K.L. Heritier, P. Henri, X. Vallières, M. Galand, E. Odelstad, A.I. Eriksson, F.L. Johansson, K. Altwegg, E. Behar, A. Beth, T.W. Broiles, J.L. Burch, C.M. Carr, E. Cupido, H. Nilsson, M. Rubin, E. Vigren, Vertical structure of the near-surface expanding ionosphere of comet 67P probed by Rosetta. Mon. Not. R. Astron. Soc. 469(Suppl 2), S118-S129 (2017). https://doi.org/10.1093/mnras/stx1459

S.M. Imber, J. Slavin, MESSENGER observations of magnetotail loading and unloading: implications for substorms at Mercury. J. Geophys. Res. Space Phys. 122(11), 11-402 (2017)

M.K. James, E.J. Bunce, T.K. Yeoman, S.M. Imber, H. Korth, A statistical survey of ultralow-frequency wave power and polarization in the Hermean magnetosphere. J. Geophys. Res. Space Phys. 121(9), 8755-8772 (2016)

R. Jarvinen, M. Alho, E. Kallio, T. Pulkkinen, Ultra-low frequency waves in the ion foreshock of Mercury: a global hybrid modeling study. Mon. Not. R. Astron. Soc. (2019)

T. Karlsson, The acceleration region of stable auroral arcs, in Chapman Conference on the Relationship Between Auroral Phenomenology and Magnetospheric Processes, Feb. 27-Mar. 04, 2011, Fairbanks, AK, American Geophysical Union (AGU) (2012), pp. 227-239

T. Karlsson, E. Liljeblad, A. Kullen, J.M. Raines, J.A. Slavin, T. Sundberg, Isolated magnetic field structures in Mercury's magnetosheath as possible analogues for terrestrial magnetosheath plasmoids and jets. Planet. Space Sci. 129, 61-73 (2016)

Y. Kasaba, J.L. Bougeret, L. Blomberg, H. Kojima, S. Yagitani, M. Moncuquet, J.G. Trotignon, G. Chanteur, A. Kumamoto, Y. Kasahara, J. Lichtenberger, Y. Omura, K. Ishisaka, H. Matsumoto, The plasma wave investigation (PWI) onboard the BepiColombo/MMO: first measurements of electric fields, electromagnetic waves, and radio waves around Mercury. Space Sci. Rev. 56, 238-278 (2010). https://doi.org/10.1016/j.pss.2008.07.017

Y. Kasaba, K. Ishisaka, Y. Kasahara, T. Imachi, S. Yagitani, H. Kojima, S. Matsuda, M. Shoji, S. Kurita, T. Hori, A. Shinbori, M. Teramoto, Y. Miyoshi, T. Nakagawa, N. Takahashi, Y. Nishimura, A. Matsuoka, A. Kumamoto, F. Tsuchiya, R. Nomura, Wire probe antenna (WPT) and electric field detector (EFD) of plasma wave experiment (PWE) aboard the Arase satellite: specifications and initial evaluation results. Space Sci. Rev. 69, 174 (2017). https://doi.org/10.1186/s40623-017-0760-X

Y. Kasaba, H. Kojima, M. Moncuquet, J.E. Wahlund, S. Yagitani, F. Sahraoui, P. Henri, T. Karlsson, Y. Kasahara, A. Kumamoto et al., Plasma wave investigation (PWI) aboard BepiColombo Mio on the trip to the first measurement of electric fields, electromagnetic waves, and radio waves around Mercury. Space Sci. Rev. 216, 65 (2020). https://doi.org/10.1007/s11214-020-00692-9

Y. Kasahara, Y. Kasaba, H. Kojima, S. Yagitani, K. Ishisaka, A. Kumamoto, F. Tsuchiya, M. Ozaki, S. Matsuda, T. Imachi, Y. Miyoshi, M. Hikishima, Y. Katoh, M. Ota, M. Shoji, A. Matsuoka, I. Shinohara, The plasma wave experiment (PWE) on board the Arase (ERG) satellite. Earth Planets Space 70, 86 (2018). https://doi.org/10.1186/s40623-017-0759-3

Y.V. Khotyaintsev, P.A. Lindqvist, C.M. Cully, A.I. Eriksson, M. André, In-flight calibration of double-probe electric field measurements on cluster. Geosci. Instrum. Method. Data Syst. 3(2), 143-151 (2014)

H. Laakso, A. Pedersen, Ambient electron density derived from differential potential measurements. Geophys. Monogr. 102, 49-54 (1998)

G. Le, P.J. Chi, X. Blanco-Cano, S. Boardsen, J.A. Slavin, B.J. Anderson, H. Korth, Upstream ultra-low frequency waves in Mercury's foreshock region: MESSENGER magnetic field observations. J. Geophys. Res. Space Phys. 118(6), 2809-2823 (2013)

W. Li, M. André, Y.V. Khotyaintsev, A. Vaivads, D.B. Graham, S. Toledo-Redondo, C. Norgren, P. Henri, C. Wang, B.B. Tang, B. Lavraud, Y. Vernisse, D.L. Turner, J. Burch, R. Torbert, W. Magnes, C.T. Russell, J.B. Blake, B. Mauk, B. Giles, C. Pollock, J. Fennell, A. Jaynes, L.A. Avanov, J.C. Dorelli, D.J. Gershman, W.R. Paterson, Y. Saito, R.J. Strangeway, Kinetic evidence of magnetic reconnection due to Kelvin-Helmholtz waves. Geophys. Res. Lett. 43(11), 5635-5643 (2016). https://doi.org/10.1002/ 2016GL069192

E. Liljeblad, T. Karlsson, Investigation of 20-40 MHz ULF waves and their driving mechanisms in Mercury's dayside magnetosphere. Ann. Geophys. 35, 879-884 (2017)

E. Liljeblad, T. Karlsson, T. Sundberg, A. Kullen, Observations of magnetospheric ULF waves in connection with the Kelvin-Helmholtz instability at Mercury. J. Geophys. Res. Space Phys. 121(9), 8576-8588 (2016) 
P.A. Lindqvist, G. Olsson, R. Torbert, B. King, M. Granoff, D. Rau, G. Needell, S. Turco, I. Dors, P. Beckman et al., The spin-plane double probe electric field instrument for MMS. Space Sci. Rev. 199(1-4), 137165 (2016)

I. Mann, Comets as a possible source of nanodust in the solar system cloud and in planetary debris discs. Philos. Trans. R. Soc. A, Math. Phys. Eng. Sci. 375(2097), 20160254 (2017)

H. Matsumoto, I. Nagano, R.R. Anderson, H. Kojima, K. Hashimoto, M. Tsutsui, T. Okada, I. Kimura, Y. Omura, M. Okada, Plasma wave observations with geotail spacecraft. J. Geomagn. Geoelectr. 46, 59-95 (1994)

H. Matsumoto, T. Okada, K. Hashimoto, I. Nagano, S. Yagitani, M. Tsutsui, Y. Kasaba, K. Tsuruda, H. Hayakawa, A. Matsuoka, S. Watanabe, H. Ueda, Y. Kasahara, Y. Omura, K. Ishisaka, T. Imachi, Y. Tateno, Low Frequency plasma wave Analyzer (LFA) onboard the PLANET-B spacecraft. Earth Planets Space 50(3), 223-228 (1998). https://doi.org/10.5636/jgg.46.59

N. Meyer-Vernet, M. Maksimovic, A. Czechowski, I. Mann, I. Zouganelis, K. Goetz, M. Kaiser, O.S. Cyr, J.L. Bougeret, S. Bale, Dust detection by the wave instrument on STEREO: nanoparticles picked up by the solar wind? Sol. Phys. 256(1-2), 463-474 (2009)

A. Milillo, M. Fujimoto, G. Murakami, J. Benkhoff, J. Zender, S. Aizawa, M. Dósa, L. Griton, D. Heyner, G. Ho et al., Investigating Mercury's environment with the two-spacecraft BepiColombo mission. Space Sci. Rev. 216(5), 1-78 (2020)

A. Milillo, M. Fujimoto, E. Kallio, S. Kameda, F. Leblanc, Y. Narita, G. Cremonese, H. Laakso, M. Laurenza, S. Massetti, S. McKenna-Lawlor, A. Mura, R. Nakamura, Y. Omura, D. Rothery, K. Seki, M. Storini, P. Wurz, W. Baumjohann, E. Bunce, Y. Kasaba, J. Helbert, A. Sprague (Hermean Environment WG Members), The BepiColombo mission: an outstanding tool for investigating the Hermean environment. Planet. Space Sci. 58, 40-60 (2010). https://doi.org/10.1016/j.pss.2008.06.005

G. Murakami, H. Hayakawa, H. Ogawa, S. Matsuda, T. Seki, Y. Kasaba, Y. Saito, I. Yoshikawa, M. Kobayashi, W. Baumjohann et al., Mio-first comprehensive exploration of Mercury's space environment: mission overview. Space Sci. Rev. 216(7), 1-25 (2020)

A. Pedersen, F. Mozer, G. Gustafsson, Electric field measurements in a tenuous plasma with spherical double probes, in Measurement Techniques in Space Plasmas: Fields, Geophys. Monogr., vol. 103 (1998), pp. 1-12. https://doi.org/10.1002/9781118664391.ch1

C. Russell, ULF waves in the Mercury magnetosphere. Geophys. Res. Lett. 16(11), 1253-1256 (1989)

J. Slavin et al., MESSENGER observations of large flux transfer events at Mercury. Geophys. Res. Lett. 37, L02105 (2010)

J.A. Slavin, S.M. Imber, S.A. Boardsen, G.A. DiBraccio, T. Sundberg, M. Sarantos, T. Nieves-Chinchilla, A. Szabo, B.J. Anderson, H. Korth et al., MESSENGER observations of a flux-transfer-event shower at Mercury. J. Geophys. Res. Space Phys. 117(A12), A00M06 (2012)

J. Slavin et al., MESSENGER observations of Mercury's dayside magnetosphere under extreme solar wind conditions. J. Geophys. Res. Space Phys. 119, 2 (2014)

J. Slavin, H. Middleton, J. Raines, X. Jia, J. Zhong, W.J. Sun, S. Livi, S. Imber, G.K. Poh, M. Akavan-Tafti et al., MESSENGER observations of disappearing dayside magnetosphere events at Mercury. J. Geophys. Res. Space Phys. (2019)

G. Stenborg, J.R. Stauffer, R.A. Howard, Evidence for a circumsolar dust ring near Mercury's orbit. Astrophys. J. 868(1), 74 (2018)

W.J. Sun, J.A. Slavin, S. Fu, J.M. Raines, Q.G. Zong, S.M. Imber, Q. Shi, Z. Yao, G. Poh, D.J. Gershman et al., MESSENGER observations of magnetospheric substorm activity in Mercury's near magnetotail. Geophys. Res. Lett. 42(10), 3692-3699 (2015)

W. Sun, J. Raines, S. Fu, J. Slavin, Y. Wei, G. Poh, Z. Pu, Z. Yao, Q. Zong, W. Wan, MESSENGER observations of the energization and heating of protons in the near-Mercury magnetotail. Geophys. Res. Lett. 44(16), 8149-8158 (2017)

T. Sundberg, S.A. Boardsen, J.A. Slavin, B.J. Anderson, H. Korth, T.H. Zurbuchen, J.M. Raines, S.C. Solomon, MESSENGER orbital observations of large-amplitude Kelvin-Helmholtz waves at Mercury's magnetopause. J. Geophys. Res. Space Phys. 117(A4), A04216 (2012). https://doi.org/10.1029/ 2011JA017268

T. Sundberg, S.A. Boardsen, J.A. Slavin, V.M. Uritsky, B.J. Anderson, H. Korth, D.J. Gershman, J.M. Raines, T.H. Zurbuchen, S.C. Solomon, Cyclic reformation of a quasi-parallel bow shock at Mercury: MESSENGER observations. J. Geophys. Res. Space Phys. (2013)

M.B. Syal, P.H. Schultz, M.A. Riner, Darkening of Mercury's surface by cometary carbon. Nat. Geosci. 8(5), 352 (2015)

K. Tsuruda, H. Hayakawa, M. Nakamura, T. Okada, A. Matsuoka, F.S. Mozer, R. Schmidt, Electric field measurements on the geotail satellite. J. Geomagn. Geoelectr. 46, 693-711 (1994). https://doi.org/10.5636/ jgg.46.693 
P. Wurz, D. Gamborino, A. Vorburger, J. Raines, Heavy ion composition of Mercury's magnetosphere. J. Geophys. Res. Space Phys. 124(4), 2603-2612 (2019)

S. Yagitani, M. Ozaki, F. Sahraoui, L. Mirioni, M. Mansour, G. Chanteur, C. Coillot, S. Ruocco, V. Leray, M. Hikishima et al., Measurements of magnetic field fluctuations for plasma wave investigation by the search coil magnetometers (SCM) onboard Bepicolombo Mio (Mercury Magnetospheric Orbiter). Space Sci. Rev. 216(7), 1-26 (2020)

H. Yamakawa, H. Ogawa, Y. Sone, H. Hayakawa, Y. Kasaba, T. Takashima, T. Mukai, T. Tanaka, M. Adachi, BepiColombo Mercury magnetospheric orbiter design. Acta Astronaut. 62, 699-705 (2008). https://doi.org/10.1016/j.actaastro.2008.01.040

A. Zaslavsky, N. Meyer-Vernet, I. Mann, A. Czechowski, K. Issautier, G. Le Chat, F. Pantellini, K. Goetz, M. Maksimovic, S. Bale et al., Interplanetary dust detection by radio antennas: mass calibration and fluxes measured by STEREO/WAVES. J. Geophys. Res. Space Phys. 117(A5), A05102 (2012) 